\title{
Therapy of Primary Ciliary Dyskinesia
}

\section{Workshop „Primäre Ziliendyskinesie“}

Thomas Nüßlein, Universitätskinderklinik Bochum

Die primäre Ziliendyskinesie (PCD) ist eine seltene Erkrankung. Therapiestudien mit betroffenen Patienten wurden bisher kaum durchgeführt, sodass es in der wissenschaftlichen Fachliteratur nur wenige verwertbare Angaben zur Behandlung gibt. Der behandelnde Arzt ist daher überwiegend auf eigene Überlegungen und Erfahrungen angewiesen. Auch dies gestaltet sich jedoch schwierig, da die meisten Ärzte nur wenige Patienten mit PCD betreuen. Ein Ziel dieser Tagung war daher, die aus verschiedenen Quellen verfügbaren Erkenntnisse zu sammeln und in komprimierter Form darzustellen.

Die Bochumer Ambulanz für Primäre Ziliendyskinesie organisierte mit Unterstützung des Beirates der Patientenorganisation „Kartagener-Syndrom und Primäre Ciliäre Dyskinesie e.V.“ einen Workshop. Er hatte zwei Schwerpunkte: das Sammeln der vorhandenen Daten und das Erarbeiten eines Therapiekonzeptes. Dabei sollte es ausschließlich um die Therapie der AtemwegsManifestationen gehen. Zusätzlich hatten die Teilnehmer Gelegenheit, einen Vortrag zum Spannungsfeld zwischen Evidenzbasierter Medizin und Einzelfallbeobachtung zu hören.

\section{Das Krankheitsbild der Primären Ziliendyskinesie}

Horst Seithe, Klinik für Kinder und Jugendliche, Klinikum Nürnberg Süd

Die primäre Ziliendyskinesie ist eine autosomal rezessive Erkrankung mit einer Häufigkeit von rund 1 zu 20000. Nur einige wenige Mutationen wurden bisher identifiziert; man untersucht derzeit rund 300 Kandidatengene mit einem Schwerpunkt auf den Chromosomen 5 und 6. In Deutschland müsste es rechnerisch etwa 4000 Patienten mit dieser Erkrankung geben, davon etwa 900 Kinder und Jugendliche. Pro Jahr kommen in Deutschland etwa 50 Neugeborene mit dieser Störung auf die Welt. Der Selbsthilfeverein „Kartagener-Syndrom und Primäre Ciliäre Dyskinesie e.V.“ überblickt derzeit etwa 200 Patienten. Am Olgahospital in Stuttgart befindet sich ein Patientenregister im Aufbau.

Die Diagnose wird bei etwa einem Drittel der Patienten im Kindes- und Jugendalter gestellt. Bei Neugeborenen und Säuglingen führt häufig ein Situs inversus zur Diagnose. In dieser Altersgruppe kommt außerdem ein unerklärbares Atemnotsyndrom bei normal entbundenen Neugeborenen vor, es gibt rezidivierende Pneumonien, persistierenden Husten, rezidivierende Rhinitiden und einen geringgradigen Hydrocephalus. Zusätzlich können Herzfehler und andere Missbildungen bestehen. Die bei Kindern und Jugendlichen dominierende Symptomatik beinhaltet rezidivierende Rhinosinusitiden, obstruktive Lungenerkrankungen, Bronchitis, Pneumonien sowie Otitiden, auch mit Hörmin- 
derung. Weitere mögliche Manifestationen sind Glaukom, Myopie, Iriskolobom und Gastroösophagealer Reflux. Im Erwachsenenalter nehmen die Otitiden $a b$, man findet dann $u$. U. eine Tympanosklerose oder ein Cholesteatom. Von pulmonaler Seite ist eine schwere chronische Bronchitis häufig. Bei Männern fällt die Hypofertilität auf, bei Frauen kommt es häufiger zu extrauterinen Schwangerschaften [1]. Zur Häufigkeit dieser Manifestationen gibt es in der Literatur nur spärliche Angaben. Hoch verdächtig für eine primäre Ziliendyskinesie sind rezidivierende Otitiden, Sinusitiden und Pneumonien mit und ohne Situs inversus.

Die Langzeitfolgen der primären Ziliendyskinesie können erheblich sein. Bedeutsam für die Prognose ist vor allem die Lungenbeteiligung mit schweren eitrigen Bronchitiden, Bronchiektasien, Atelektasen, dauerhafter Besiedlung mit Pseudomonas aeruginosa, Hämoptysen oder Pneumothoraces. Auch eine beeinträchtigende Hörminderung kommt häufig vor. Im Endstadium kommt es zur respiratorischen Insuffizienz. Viele Betroffene werden vorzeitig berentet.

Bei der Betreuung der Patienten kommt es darauf an, eine enge Zusammenarbeit zwischen dem pneumologisch versierten Arzt und einem HNO-Arzt zu etablieren. Ähnlich wie bei der Mukoviszidose werden regelmäßige Ambulanzbesuche alle 3-4 Monate empfohlen. Vorstellungen beim HNO-Arzt sind zweimal jährlich erforderlich. Routineuntersuchungen wie Lungenfunktionsdiagnostik, Röntgen-Thorax-Aufnahmen und Blutentnahmen sollten nach einem festen Schema in regelmäßigen Intervallen erfolgen.

\section{Sekretmobilisation und Sekretolyse}

für die unteren Atemwege

Claudia Suerbaum, Kinderklinik am Marien-Hospital Wesel

Der Zilienschlag erfolgt normalerweise koordiniert. Die Zilien schlagen zunächst aufgerichtet antegrad, krümmen sich dann und bewegen sich anschließend nach retrograd in die Ausgangsposition zurück. Dadurch werden inhalierte Partikel aus den peripheren Atemwegen oralwärts abtransportiert. Diese Abfolge ist bei Ziliendyskinesie nicht gegeben, und die mukoziliäre Clearance ist dadurch erheblich beeinträchtigt [2].

Therapeutische Interventionen zielen darauf hin, diese defekte Reinigungsfunktion auszugleichen. Grundsätzlich dazu geeignet sind Physiotherapie, Bronchodilatatoren und Mukolytika. Die Physiotherapie hat einen hohen Stellenwert und sollte zweimal täglich für etwa 20 Minuten durchgeführt werden. In einer aktuellen Publikation empfehlen Bush und Mitarb. [3] für größere Kinder, Jugendliche und Erwachsene als geeignete Technik jeweils vier betonte Inspirationen hintereinander und ein daran anschließendes forciertes Exspirationsmanöver. Durch entsprechende Lagerung des Patienten sollte die Schwerkraft ausgenutzt werden. Bei Exazerbationen sind häufigere physiotherapeutische Anwendungen nötig. Eine enge Anbindung des Patienten an eine auf Atemphysiotherapie spezialisierte krankengymnastische Praxis ist empfehlenswert. Außerdem sollten PCD-Patienten dazu angehalten werden, regelmäßig Sport zu treiben. Körperli- che Aktivität sei der beste Bronchodilatator, so die Referentin. Medikamentös können $\beta_{2}$-Sympathomimetika bei vielen Patienten hilfreich sein, jedoch muss die individuelle Reaktion im Broncholysetest verifiziert werden. So zeigte sich in einer vergleichenden Studie bei 12 Patienten mit primärer Ziliendyskinesie, dass durch körperliche Aktivität in Form einer Laufbandbelastung eine signifikante Verbesserung der Lungenfunktion erreicht werden konnte, nicht jedoch durch Inhalation von $200 \mu \mathrm{g}$ Salbutamol [4]. Bei der Verordnung von Mukolytika ist ganz allgemein zu berücksichtigen, dass bei schwerer betroffenen Patienten eine starke Verflüssigung des Schleims vorübergehend zu einer Verschlechterung führen kann, wenn das Sekret nicht ausreichend abgehustet wird und in die Lungenperipherie läuft. Gesicherte Daten zu Mukolytika bei PCD gibt es nicht. Orale Mukolytika können unter Umständen hilfreich sein, beispielsweise Ambroxol oder N-Acetylcystein. Das A und O der Sekretmobilisation bei primärer Ziliendyskinesie seien Sport und Physiotherapie, schloss Suerbaum.

\section{Selkretmobilisation und Sekretolyse für die oberen Atemwege}

Thomas Nüßlein, Universitätskinderklinik Bochum

Zur Sekretmobilisation in den oberen Atemwegen stehen verschiedene Medizinprodukte zur Verfügung. Als mechanisches Verfahren zum Reinigen der Nase hat sich die Nasendusche etabliert. Dazu wird ein spezielles Kännchen benutzt, das z.B. mit Emser Salz als Therapeutikum gefüllt wird (Abb.1). Das elektrische Gerät Pari Sole produziert erwärmte Flüssigkeitströpfchen, die so groß sind, dass sie vorwiegend in den oberen Atemwegen deponiert werden. Von derselben Firma wurde auch ein neues Gerät speziell für die Behandlung der Nasennebenhöhlen entwickelt, das Pari Sinus, das jedoch noch nicht im Handel ist. Es übt einen erhöhten Druck auf die oberen Atemwege aus, in dem mit Hilfe eines zweiten Schlauchsystems eine sinusförmige Welle erzeugt wird, die die Inhalation überlagert. Auf diese Weise gelang das inhalierte Aerosol bis in die Nasennebenhöhlen.

Als Wirkstoffe für die Sekretmobilisation im Bereich der oberen Atemwege eignen sich grundsätzlich systemische Sekretolytika, inhalierbare Salzlösungen und topische Sekretolytika. Sucht man zu diesen Medikamentengruppen wissenschaftliche Fachliteratur mit Hilfe einer PubMed-Recherche, so findet man weder Cochrane-Reviews noch andere Meta-Analysen, Originalarbeiten oder Reviews. Das Suchwort „chronische Rhinosinusitis“ ist ergiebiger. So findet man beispielsweise ein Modell zur Pathogenese der Bronchiektasie, das durchaus Parallelen zur Situation in der Nase aufweist. Auch hier führt das Wechselspiel zwischen Inflammation und gestörter mukoziliärer Clearance zum Untergang von Gewebe. Ein wesentlicher Unterschied zur zystischen Fibrose besteht darin, dass die periziliäre Flüssigkeit bei der PCD nicht verändert ist. Dadurch ist der Mukus durch Husten viel leichter zu mobilisieren als bei der Mukoviszidose.

Als realistisches Therapieziel bezeichnete Nüßlein eine bessere Mobilisation des Sekrets mit der Folge einer geringeren Sekreteretention. Im Unterschied dazu wird ist es viel schwieriger, die Sekretbildung zu beeinflussen. 


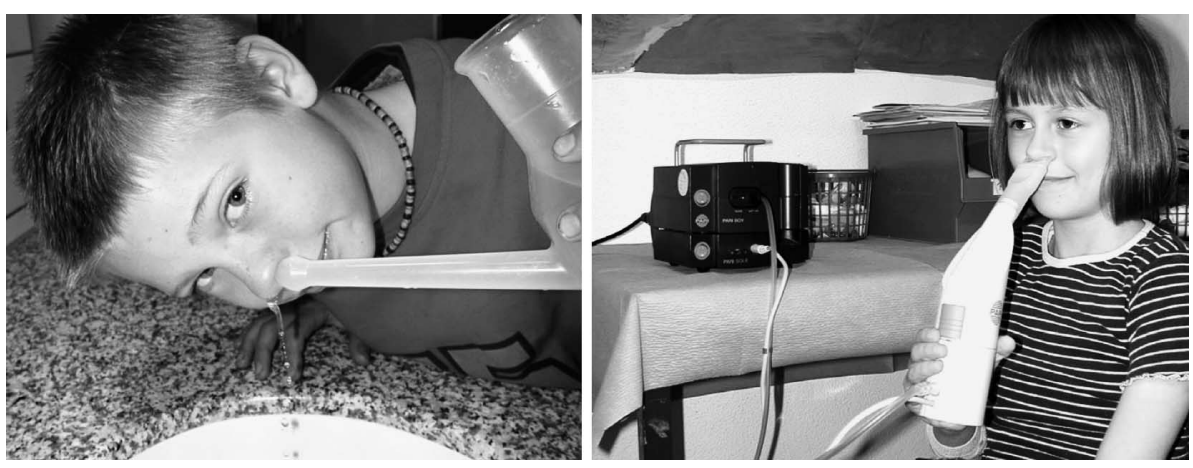

Abb. 1 Durchführung der Nasendusche (links) und Anwendung des Pari-SoleGerätes (rechts).

Zur chronischen Sinusitis liegen mehrere Therapiestudien vor, die der Referent kurz zusammenfasste. Nasenspülungen mit hypertoner Kochsalzlösung führten bei 150 Erwachsenen mit chronischer Sinusitis zu einer Reduktion der üblichen Therapie um 35\% [5]. Ein Vergleich zwischen Nasendusche und Nasenspray mit Meerwasser ergab günstigere endoskopische Befunde nach Nasendusche und eine bessere Lebensqualität nach Nasenspray [6]. Dass hypertone Kochsalzlösung als Nasendusche eine Wirkung hat, zeigte auch eine randomisierte, prospektive Studie bei 74 Erwachsenen mit Sinusitis [7]. Nach sechsmonatiger Behandlung war der „Rhinosinusitis Disability Index“ in der VerumGruppe signifikant besser als zu Beginn, während er bei den nicht behandelten Patienten keine Veränderung aufwies. Auch zur topischen Anwendung von Antibiotika gibt es Angaben in der Literatur. Die Anwendung von Tobramycin über vier Wochen wurde mit der Inhalation von Salzlösung verglichen, wobei ein Vernebler für große Tröpfchen verwendet wurde [8]. In beiden Gruppen kam es zu einer Verbesserung von Symptomen, Lebensqualität und Schleimhautbefunden. Unter Tobramycin erfuhren die Patienten eine schnellere Schmerzlinderung, hatten aber mehr nasalen Ausfluss. Beide Therapieformen führten nur selten zu Nebenwirkungen. Bei 30 Kindern mit chronischer Sinusitis erfolgte ein doppelblinder Vergleich zwischen physiologischer und hypertoner Kochsalzlösung [9]. Den Behandlungserfolg erfasste man mit Hilfe verschiedener Scores unter Einbeziehung der radiologischen Befunde. Nach vierwöchiger Behandlung mit 3,5-\%iger Kochsalzlösung hatten sich alle Scores signifikant verbessert. Kinder, die mit physiologischer Kochsalzlösung inhaliert hatten, verbesserten sich nur beim „postnasal drip-Score“. Auch vier Wochen nach Beendigung der Therapie fanden sich nachhaltige Verbesserungen.

Bei kritischer Analyse dieser publizierten Daten muss man feststellen, dass die meisten Studien nur wenige Patienten umfassten, dass die Populationen insgesamt heterogen waren, dass überwiegend Erwachsene behandelt wurden und die wenigsten Studien randomisiert, kontrolliert oder sogar doppelblind durchgeführt wurden. Hinzu kommt das generelle Problem, dass Patienten mit primärer Ziliendyskinesie nicht gesondert untersucht wurden. Dennoch zeigen diese Ergebnisse, dass eine lokale sekretmobilisierende Behandlung oft wirksam ist, auch wenn die Responseraten nur bei rund 50\% liegen. Außerdem scheinen die verwendeten Wirkstoffe den Therapieerfolg zu beeinflussen.

Das Vorgehen in der eigenen Klinik fasste Nüßlein in einem Flussdiagramm zusammen (Abb. 2). Bei chronischer Rhinosinusitis besteht die Basisbehandlung in der Anwendung der Nasen- dusche. Ist diese allein nicht erfolgreich, wird das Pari Sole eingesetzt. Beim individuellen Patienten muss entschieden werden, ob zusätzlich eine antientzündliche oder antibiotische Behandlung erforderlich ist. Es gibt noch zahlreiche offene Fragen, die in Studien beantwortet werden müssen. Unklar ist, wann eine Behandlung begonnen werden muss, welche Zielparameter den Behandlungserfolg am besten erfassen, ob die Behandlung wieder beendet werden kann und welches die wirksamsten Medikamente sind. Als Methode für die systematische Erfassung des Therapieerfolgs schlug Nüßlein die $\mathrm{n}=1$-Studie vor.

\section{Antientziindliche Therapie}

Hans-Georg Bresser und Kerstin Landwehr, Kinderklinik der Krankenanstalten Gilead, Bielefeld

Auf der Suche nach wissenschaftlichen Grundlagen zur antiinflammatorischen Behandlung bei PCD hatten die Autoren zunächst eine Medline-Recherche durchgeführt. Dabei kam man jedoch zu einem negativen Ergebnis. Im nächsten Schritt wurden Lehrbücher der pädiatrischen Pneumologie konsultiert. In der letzten Auflage des Lehrbuches von Chernick und Boat, „Disorders of the Respiratory Tract in Children“, wird der Stellenwert der antientzündlichen Therapie bei PCD als ungeklärt bezeich-

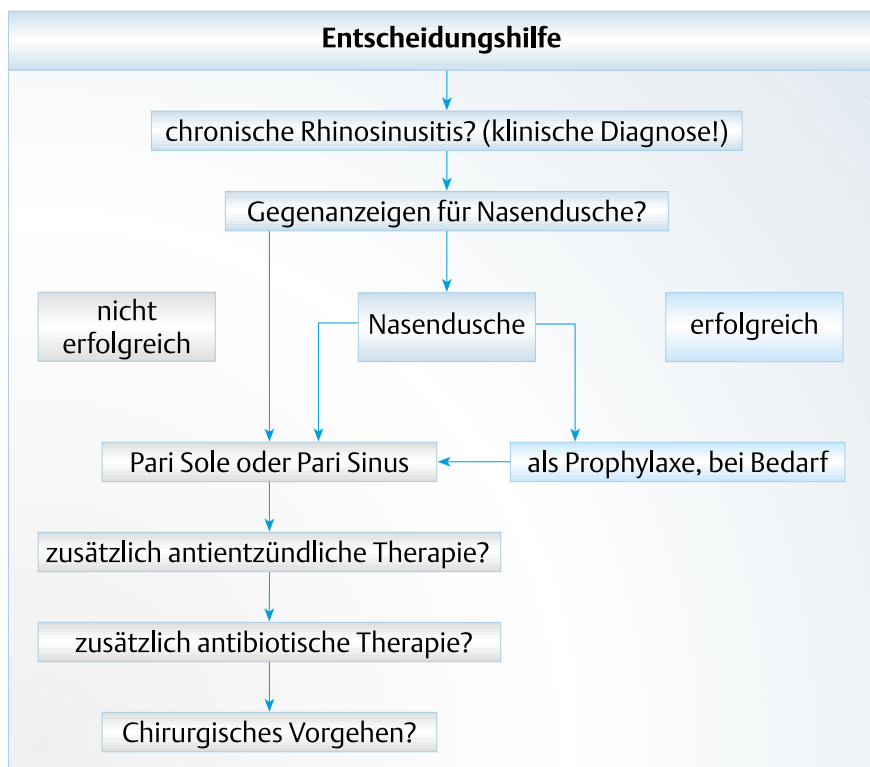

Abb. 2 Flussdiagramm zur Therapie der Rhinosinusitis. 
net. In Textbüchern von Taussig und Landau oder von Nelson wird lediglich erwähnt, dass es keine spezifische Therapie für die Ziliendyskinesie gibt und dass die Behandlung symptomatisch ist.

Nachdem man die Perspektive etwas erweitert hatte, fand man einige indirekte Quellen zur antientzündlichen Therapie. So bestand bei PCD-Patienten keine Beziehung zwischen NO-Konzentrationen in Nase und Lunge und einer Therapie mit Steroiden, und die NO-Konzentrationen korrelierten auch nicht mit der Lungenfunktion [10]. In einer Arbeit über den Nutzen von $\beta_{2}$-Sympathomimetika gab es zwar Hinweise für eine obstruktive Lungenerkrankung bei Kindern mit PCD, jedoch keinen klaren Nachweis der Wirksamkeit der antiobstruktiven Behandlung [4].

Zusammengefasst lässt sich nach Auswertung der Fachliteratur keine klare Aussage zur Wirksamkeit einer antientzündlichen Therapie bei primärer Ziliendyskinesie treffen.

Topische antibiotische Therapie für die oberen

und unteren Atemwege

Silke van Koningsbruggen, Universitätskinderklinik Köln

Das Ziel einer topischen Antibiotikatherapie sollte sein, Komplikationen der Erkrankung zu vermeiden und eine Zunahme der Symptomatik zu verhindern. Zum Nutzen einer lokalen Antibiotikabehandlung fanden sich bei einer Medline-Recherche keine passenden Literaturstellen. Im Lehrbuch Pädiatrische Pneumologie [11] von Rieger und Mitarb. wird die antibiotische Prophylaxe und die aggressive antibiotische Therapie von Infektionen als therapeutische Möglichkeit bei Ziliendyskinesie erwähnt. Im Übersichtsartikel von Bush [3] findet sich ein ähnlicher Hinweis zum Nutzen einer aggressiven Antibiotikabehandlung, die topische bzw. inhalative Applikation wird jedoch nicht explizit erwähnt.

Die Referentin beschäftigte sich daher mit der Frage, ob die zur Mukoviszidose (Zystische Fibrose, $\mathrm{CF}$ ) verfügbaren Daten über inhalative Antibiotika auch für die primäre Ziliendyskinesie anwendbar sind. In einem Cochrane-Review wurden plazebokontrollierte, randomisierte Studien zusammengefasst, in denen bei CF über mindestens vier Wochen ein topisches Antibiotikum inhaliert worden war. In neun Studien besserte das Antibiotikum, meist Tobramycin, die Einsekundenkapazität in signifikant stärkerem Ausmaß als Plazebo. Bei einem direkten Vergleich der Antibiotika Tobramycin und Colistin fand man eine etwas stärkere Verbesserung der $\mathrm{FEV}_{1}$ unter Tobramycin [12]. Wie lange der günstige Effekt einer inhalativen Applikation anhält, in welchem Ausmaß Resistenzentwicklungen klinisch problematisch werden, und inwiefern auf lange Sicht Nebenwirkungen ernsthafter Natur auftreten, bleibt bis heute unklar. Es besteht auch keine ausreichende Evidenz bezüglich der Wahl des Antibiotikums und der zu verabreichenden Dosis.

Auch von erwachsenen Patienten mit Bronchiektasen liegen Ergebnisse zur topischen Antibiotikatherapie vor. In einer doppelblinden, plazebokontrollierten Studie führte die kurzzeitige Inhalation von Tobramycin zu klinischen Verbesserungen, zu einer geringeren Pseudomonas-Dichte und bei 13 der 37 Patienten sogar zur Eradikation von Pseudomonas aeruginosa [13]. Unter Plazebo verschwanden die Bakterien bei keinem der 35 Patienten. Bei der Entwicklung der Einsekundenkapazität ergaben sich keine Unterschiede zwischen den Gruppen. Stationäre Aufenthalte waren unter Tobramycin bei fünf Patienten, unter Plazebo nur bei einem Patienten erforderlich. Eine andere plazebokontrollierte Doppelblindstudie untersuchte die Inhalation von Tobramycin bei Patienten mit Bronchiektasen [14]. Das Antibiotikum hatte günstige Auswirkungen auf die Sputummenge, die Viskosität des Sputums, den Peak flow, die Sechs-Minuten Gehstrecke und die nächtliche Sauerstoff-Sättigung. Außerdem fand man eine Abnahme der Konzentrationen von Myeloperoxidase im Sputum. In einer anderen Studie wurden Patienten mit Bronchiektasen und chronischer P. aeruginosa-Infektion im Anschluss an eine intravenöse Antibiotikabehandlung täglich zweimal mit einer Kombination aus inhalativem Ceftazidim (Einzeldosis $1000 \mathrm{mg}$ ) und Tobramycin (Einzeldosis $100 \mathrm{mg}$ ) behandelt [15]. Nach einer antibiotischen Behandlung über 12 Monate fand man im Vergleich zur Plazebogruppe eine reduzierte Zahl stationärer Aufenthalte (0,6 vs. 2,5) und eine geringere Zahl von Tagen, die im Krankenhaus verbracht werden mussten (13,1 vs. 57,9).

Diese Studien weisen darauf hin, dass auch bei primärer Ziliendyskinesie inhalative Antibiotika für bestimmte Patienten sinnvoll sein können. Es lassen sich jedoch keine Angaben zur Indikation ableiten. Ebenso wenig klar ist, welches die günstigste Substanz ist, welche Dosierung erforderlich ist und wie lange behandelt werden muss.

In der Diskussion wurde vorgeschlagen, bei pulmonalen Exazerbationen eine intravenöse Antibiotikatherapie durchzuführen. Dabei müsse man jedoch berücksichtigen, dass Pseudomonaden bei primärer Ziliendyskinesie nicht die dominanten Keime in der Lunge sind. Wenn ein Patient mit Pseudomonas aeruginosa infiziert ist, sei häufig die Gabe eines oralen Chinolons wirksam. Damit ließen sich die Bakterien häufig auch über längere Zeit eradizieren. Nur wenige Patienten benötigten eine Inhalation mit hochdosiertem Tobramycin, beispielsweise bei häufigen respiratorischen Exazerbationen. Eine Besonderheit bei PCD ist die Hörminderung, die bei vielen Patienten besteht. Da oft gleichzeitig eine Trommelfell-Perforation vorliegt, ist an eine topische antibiotische Therapie des Mittelohrs zu denken. Gyrasehemmer haben diesbezüglich Vorteile gegenüber Aminoglykosiden. Bei einem Trommelfelldefekt kann man Gyrasehemmer in Form von Augentropfen verabreichen. Besteht eine Infektion des Ohres mit Pseudomonas aeruginosa, seien die Bakterien hier kaum zu eradizieren. Zum Säubern des Ohres biete sich dann 3-\%iges $\mathrm{H}_{2} \mathrm{O}_{2}$ an, und im Anschluss daran könne man das Antibiotikum ins Ohr tropfen. Allgemein könne man jedoch festhalten, dass eine prophylaktische Antibiotikatherapie bei primärer Ziliendyskinesie in der Regel oral erfolgen kann und daher keiner topischen Therapie bedarf. 
Systemische antibiotische Therapie

Jan Buschmann, Universitätskinderklinik Heidelberg

Die meisten Patienten mit primärer Ziliendyskinesie sind von bakteriellen Infektionen betroffen. Dabei geht es um drei verschiedene Organsysteme. Die chronische eitrige Otitis media betrifft mindestens $70 \%$ aller Patienten und kann zur Hörminderung und zur Sprachentwicklungsverzögerung führen. Mit mindestens $90 \%$ noch häufiger ist die chronische Rhinosinusitis. Eine chronische eitrige Lungenerkrankung wird ebenfalls bei mehr als $90 \%$ der Patienten beobachtet, wobei in 29 - $86 \%$ Bronchiektasen nachgewiesen werden. Ziel einer antibiotischen Therapie muss sein, diese Komplikationen zu verhindern (Abb. 3).

\begin{tabular}{|c|}
\hline Ziele und Strategien antibiotischer Therapie \\
\hline $\begin{array}{c}\text { Verhinderung von progressiver Lungenzerstörung } \\
\text { und Ausbildung von Bronchiektasen }\end{array}$ \\
\hline \begin{tabular}{c} 
Prompte antibiotische Therapie bei Atemwegsinfekten \\
\hline $\begin{array}{c}\text { Aggressive verlängerte hochdosierte orale antibiotische } \\
\text { Therapie bei bronchopulmonalen Exazerbationen }\end{array}$ \\
\hline $\begin{array}{c}\text { Intravenöse antibiotische Therapie bei ausbleibender } \\
\text { Besserung unter oraler Therapie und bei } \\
\text { Pseudomonas-Infektion }\end{array}$ \\
Antibiotische Langzeitprophylaxe \\
\hline Bush et al., Eur Respir/ 1998; 12:982-988 · Meeks \& Bush, Pediatr Pulmonol 2000; 29:307-316 \\
Ahrens \& Körbler, HNO aktuell 2002; 10:391-400
\end{tabular} \\
\hline
\end{tabular}

Abb. 3 Ziele der systemischen Antibiotikatherapie der pulmonalen Beteiligung bei primärer Ziliendyskinesie.

Um akute Exazerbationen bei Bronchiektasen zu definieren, wurde ein Punktesystem vorgeschlagen [16]. Zeichen der akuten Verschlechterung sind vermehrter Husten, veränderte Sputumproduktion, Müdigkeit, Abgeschlagenheit, verminderte Belastbarkeit, Fieber, Dyspnoe, ein veränderter Auskultationsbefund mit Rasselgeräuschen, vermehrtes Giemen, verminderte Lungenfunktion oder neu aufgetretene radiologische Veränderungen. Wenn mindestens 4 dieser 9 Beschwerden vorliegen, kann dies als akute Exazerbation gewertet werden. Hier muss man jedoch berücksichtigen, dass auch andere respiratorische Störungen, wie beispielsweise ein Asthmaanfall, eine Punktzahl von 4 ergeben können.

Bei der Auswahl des optimalen Antibiotikums ist der Erregernachweis aus dem Sputum hilfreich. Die routinemäßigen vierteljährlichen Kontrollen sollten daher auch die Sputumdiagnostik beinhalten. Nützlich sind auch Abstriche aus Ohr und Nase bei entsprechend betroffenen Patienten. Die dominierenden Bakterien sind Haemophilus influenzae (ca. 50\%) und Streptokokken (bis zu 40\%), seltener kommen Moraxella catarrhalis, Streptococcus viridans oder Klebsiellen vor [1]. Pseudomonas aeruginosa ist bei PCD in der Lunge ein sehr seltener Keim, der nur etwa 5\% aller akuten Exazerbationen verursacht. Hierin besteht ein relevanter Unterschied zur Mukoviszidose.
Auch bei Virusinfektionen ist eine Antibiotikatherapie sinnvoll, weil die Adhärenz von Bakterien erleichtert ist und pulmonale Exazerbationen häufiger vorkommen.

Zur Prophylaxe mit Antibiotika gibt es keine Studiendaten von Patienten mit PCD. Überträgt man Erfahrungen von anderen Krankheiten, so ist der Nutzen bisher nicht bewiesen. In jedem Fall ist als Infektionsprophylaxe die Einhaltung des allgemeinen empfohlenen Impfplans nötig. Außerdem sollten die Patienten gegen Pneumokokken und jährlich gegen Grippe geimpft werden.

Bei der chronischen Otitis media besteht über mindestens sechs Monate eine Sekretion durch das perforierte Trommelfell. Eine Therapiestudie bei 111 Kindern mit chronischem Paukenerguss ergab, dass eine zwölfmonatige Behandlung mit Amoxicillin im Vergleich zu Plazebo die Häufigkeit von akuten Otitiden signifikant senkte (von 1,0 auf 0,3 pro Person), und dass Ergüsse seltener wurden [17]. Das Hörvermögen wurde jedoch nicht verbessert. Eine $\beta$-Lactamase-Bildung war mit 33\% unter Antibiotika häufiger als unter Plazebo mit 22\%. Das Erregerspektrum bei chronisch eitriger Otitis media wird dominiert von Pseudomonas aeruginosa. Diese Bakterien werden je nach Datenquelle in $48-98 \%$ der Fälle nachgewiesen. Es folgen Staphylococcus aureus (etwa 22\%) und Streptococcus pneumoniae, Klebsiella pneumoniae, Proteus mirabilis und Escherichia coli mit jeweils $7-10 \%$. Auch Anaerobier kommen vor (Peptococcus spec., Bacteroides fragilis-Gruppe und Peptostreptococcen). Eine Sanierung der chronisch eitrigen Otitis media war nach zweiwöchiger intravenöser Behandlung mit Mezlocillin und Ceftazidim in 100\% erreichbar [18]. Wenn nur eine Spülung erfolgte, war der Therapieerfolg mit $8 \%$ deutlich geringer. In einem aktuellen CochraneReview wurde die lokale Behandlung mit Chinolon und Spülung als Therapie der Wahl bezeichnet [19].

Auch zur Therapie der chronischen Rhinosinusitis liegt ein Cochrane Review vor, jedoch nicht zur PCD [20]. Ergebnisse aus sechs Studien an insgesamt 562 Kindern legen nahe, dass eine 10 -14-tägige antibiotische Behandlung sinnvoll ist. Die „Number needed to treat"-Analyse ergab, dass acht Patienten behandelt werden müssen, um einen Patienten zusätzlich zu heilen.

Nach diesen Daten erscheint eine konsequente systemische antibiotische Behandlung von akuten bakteriellen Infektionen und bronchopulmonalen Exazerbationen sinnvoll, ebenso wie die systemische antibiotische Behandlung bei chronischer Rhinosinusitis. Demgegenüber ist die prophylaktische Behandlung bei Bronchitis oder bei Bronchiektasen allenfalls im Einzelfall angezeigt.

\section{Workshops zur Therapie der Atemwegsmanifestation bei PCD}

Am Nachmittag teilten sich die Teilnehmer in zwei Arbeitsgruppen auf, in denen sie eine Zusammenstellung der Therapieoptionen für die Atemwegserkrankungen bei PCD erarbeiteten. Die Ergebnisse wurden in tabellarischer Form zusammengefasst (Tab. 1 und Tab. 2). 
Tab. 1 Basistherapie der oberen Atemwege bei Patienten mit PCD. Mitglieder der Arbeitsgruppe: F. Ahrens, H. G. Bresser, Th. Deitmer, W. Kamin, Th. Nüßlein, K. Wiermann

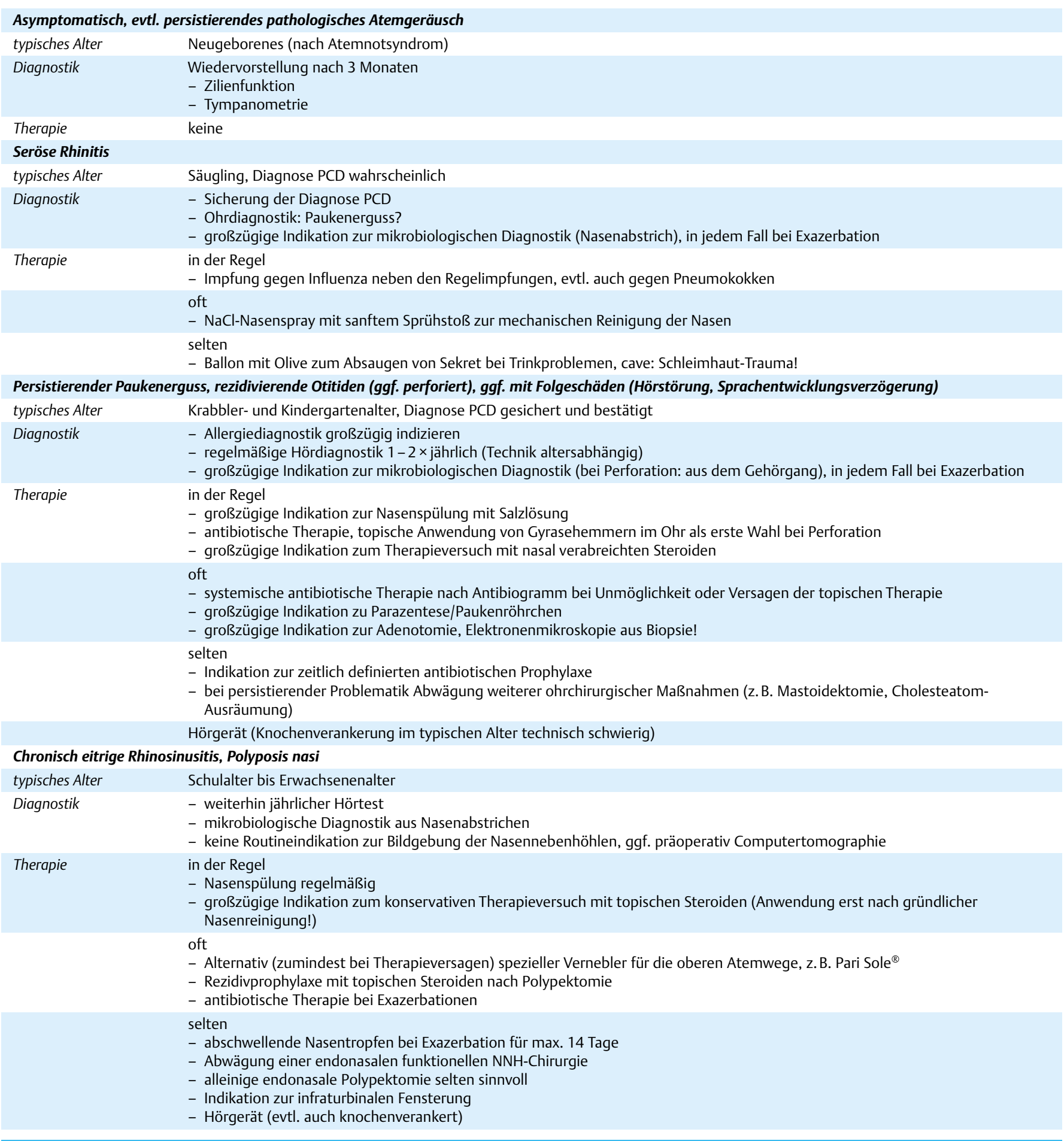

Zwischen Einzelfallbeobachtung und Metaanalyse: Therapierichtlinien im pneumologischen Alltag

Thomas Nicolai, Dr. von Haunersches Kinderspital der LudwigMaximilian-Universität München

Das Konzept der Evidenzbasierten Medizin (EBM) lässt sich in fünf Punkten zusammenfassen, die von Sackett im Jahr 1990 be- schrieben wurden (Abb. 4) [21]. Ausgangspunkt ist die Situation eines individuellen Patienten, bei dem sich eine bestimmte klinische Fragestellung ergibt. Diese Frage soll mit Hilfe der EBM beantwortet werden, indem man zunächst nach den besten verfügbaren Informationen sucht und diese dann kritisch bewertet. Dann muss geprüft werden, ob diese Erkenntnisse auf den Patienten anwendbar sind. Auch die Evaluation des eigenen Handelns gehört zur EBM. 
Tab. 2 Therapie der unteren Atemwege bei Patienten mit primärer Ziliendyskinesie: Zusammenfassung der Therapieoptionen. Mitglieder der Arbeitsgruppe: P. Ahrens, M. Barker, J. Buschmann, R. Kitz, S. van Koningsbruggen, H. Köster, K. Landwehr, C. Lex, J. Mainz, B. Niggemann, F. Panzer, S. Schmidt, H. Seithe, G. Steinkamp, C. Suerbaum

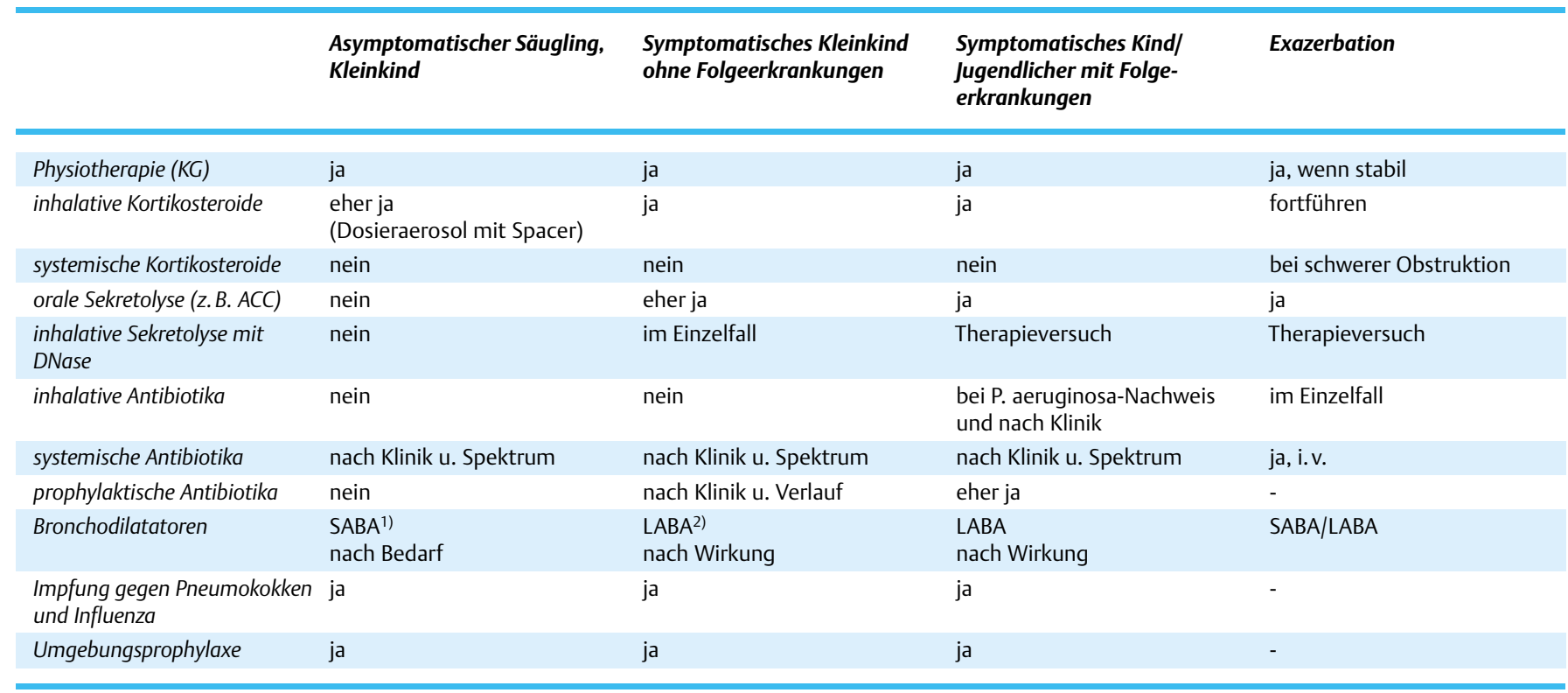

1) SABA: „short acting $\beta_{2}$-agonist“, kurzwirksames $\beta_{2}$-Sympathomimetikum

2) $L A B A$ : „long acting $\beta_{2}$-agonist“, langwirksames $\beta_{2}$-Sympathomimetikum

\begin{tabular}{|c|}
\hline \multicolumn{1}{|c|}{ Konzept EBM } \\
\hline Stellung beantwortbarer klinischer Fragen \\
\hline Suche nach der besten verfügbaren Information \\
\hline Kritische Bewertung dieser Information \\
\hline Prüfung der Anwendbarkeit auf den Patienten \\
\hline Evaluation des eigenen Handelns \\
\hline Sackett 1999 \\
\hline
\end{tabular}

Abb. 4 Konzept der Evidenzbasierten Medizin

Wenn man die vorhandenen Informationen bewertet, werden diese nach Kategorien eingeteilt, die von „Nutzen belegt“ bis „Unwirksamkeit oder Schädlichkeit wahrscheinlich“ reichen. Dabei tritt häufig das Problem auf, dass man die EBM fälschlicherweise auf randomisierte, kontrollierte Studien verkürzt und dann als unbrauchbar „verdammt“. Grenzen der EBM ergeben sich dann, wenn man auf ein Patientenproblem stößt, das nicht in klinischen Studien untersucht wurde. In diesem Zusammenhang stellen seltene Krankheiten eine besondere Herausforderung dar. Aber auch bei häufigen Erkrankungen gibt es nicht immer randomisierte kontrollierte Studien zum jeweiligen Problem. Nicolai nannte als Beispiel ein Kleinkind mit obstruktiver Bronchitis und akuter Dyspnoe, das mit Steroiden vorbehandelt ist und sich massiv gegen eine Inhalationstherapie zur Wehr setzt. Die Frage, ob intravenöses Theophyllin eine sinnvolle Therapieoption sein kann, wurde mit randomisierten kontrollierten Studien nicht untersucht.
Der Referent ließ keinen Zweifel daran, dass ohne Evidenzbasierte Medizin die Probleme noch größer wären. Erfahrungsmedizin führt zur Polypragmasie, die Kosten neuer Medikamente würden ohne EBM schwer zu rechtfertigen sein, und allgemein bestünde eine Tendenz zum Chaos bei der Therapie.

Die Evidenzbasierte Medizin bezieht nicht nur funktionelle Parameter (zum Beispiel Blutdruck oder Lungenfunktionswerte) mit in die Bewertung ein, sondern auch die Schwere der Symptome, die Lebensqualität, das Ausmaß der Behinderung oder die Gehstrecke. Wenn man den Nutzen oder Schaden neuer Behandlungen beurteilen will, bietet sich eine „Number needed to treat"-Analyse (NNT) an. Damit wird beschrieben, wie viele Patienten behandelt werden müssen, um für einen Patienten den gewünschten Nutzen zu erzielen.

Nicolai wies auf eine besondere Schwierigkeit hin, wenn randomisierte, kontrollierte Studien negative Ergebnisse zum primären Zielparameter erbracht haben. In dieser Situation wird nicht selten eine Subgruppenanalyse durchgeführt, um doch noch eine Patientengruppe herauszufiltern, die von der Behandlung profitiert. Solche „Ex post“-Analysen sind aus statistischen Gründen unzulässig. Wenn nur wenige \% der Patienten in eine solche Subgruppe gehören, kann die Rekrutierung für eine zweite Studie schwierig werden. Ein wichtiger Aspekt ist auch der richtige Zeitpunkt zur Durchführung einer klinischen Prüfung. Am Beispiel einer geplanten Studie zum Vergleich zwischen langwirksamen $\beta_{2}$-Sympathomimetika und inhalativen Steroiden bei Kindern mit Asthma und einer $\mathrm{FEV}_{1}$ unter $95 \%$ des Solls machte $\mathrm{Ni}$ colai deutlich, dass sich häufig bestimmte Behandlungen bereits in der Praxis durchgesetzt haben, bevor entsprechende Studien abgeschlossen werden, sodass dann keine geeigneten Patienten mehr rekrutiert werden können. 
Im Hinblick auf Meta-Analysen, die ein wichtiger Bestandteil der Evidenzbasierten Medizin sind, stößt man auf ganz andere Probleme. Ein „Publication Bias“ entsteht dadurch, dass vornehmlich Studien mit positiven Behandlungsergebnissen veröffentlicht werden. Häufig sind relevante Untergruppen von Patienten in den Gesamtergebnissen der Studie nicht aufgeschlüsselt, was die Anwendbarkeit der Studienergebnisse auf individuelle Patienten erschwert. Am Beispiel der Milbenprävention machte Nicolai deutlich, dass selbst Ergebnisse großer Studien an Erwachsenen nicht automatisch auf pädiatrische Patienten übertragen werden können [22]. Eine Metaanalyse zur Wirksamkeit von DNCG bei Kindern reflektierte den geschichtlichen Ablauf der Medikamenteneinführung [23]. In alten Studien waren die Ergebnisse für DNCG besser als in Studien der 1990er Jahre. Ein wichtiger Unterschied besteht darin, dass in den neueren Studien vorwiegend Kinder im Alter von 1 - 4 Jahren behandelt wurden, woraus weniger gute Behandlungserfolge resultierten, während die eigentliche Zielgruppe der Schulkinder nur in den älteren Studien untersucht worden war.

Als Beispiel für randomisierte kontrollierte Studien bei akuten Erkrankungen nannte Nicolai die Bronchiolitis bei Kindern. Im Jahr 2003 erschien eine vergleichende Studie zur Anwendung von Adrenalin vs. Plazebo [24]. Die Atemanstrengung wurde bei der ersten Behandlung durch Adrenalin zwar stärker reduziert, bei den anschließenden Behandlungszyklen fand man jedoch keinen statistisch signifikanten Unterschied zu Plazebo. Im Einzelfall kann Adrenalin dennoch eine gute Wirksamkeit besitzen, sodass für spezielle Situationen trotz EBM eine Einzelfallentscheidung erforderlich bleibt.

Die Konsequenz für den klinischen Alltag sollte sein, Einzelfallbeobachtung und EBM sinnvoll miteinander zu kombinieren. Dies gilt insbesondere für seltene akute oder chronische Erkrankungen. Hier ist eine nachvollziehbare Ergebniskontrolle unbedingt erforderlich. Eine etablierte Methode hierzu ist der „n of 1 randomised controlled trial“, der im Folgenden $n=1$-Studie genannt werden soll. Dabei wird eine randomisierte, kontrollierte Studie bei einem einzigen Patienten durchgeführt, indem die Behandlung nach Ablauf eines bestimmten Zeitraums mehrfach zwischen Verum und Plazebo hin und her gewechselt wird, beispielsweise in einem „on-off-on-off“- Schema. Als Beispiel nannte Nicolai eine kürzlich publizierte Studie zur chronischen Granulomatose, bei der die Wirksamkeit von Itraconazol überprüft wurde [25]. Man verabreichte jeweils über ein Jahr Verum bzw. Plazebo und wechselte die Behandlung von Jahr zu Jahr. Dieses Verfahren wurde nach und nach bei 39 verschiedenen Patienten durchgeführt, sodass man für die Publikation die Erfahrungen aus 120 Studienjahren zusammenfassen konnte. Während der Behandlung mit Itraconazol kam es $1 \mathrm{mal}$ zu einer AspergillusPneumonie, in den Plazebo-Jahren insgesamt 7mal. Dieser Unterschied war statistisch auf dem 5\%-Niveau signifikant.

In seinem Resümee machte Nicolai deutlich, dass Evidenzbasierte Medizin für die sorgfältige Patientenbetreuung unverzichtbar ist. Unbedingt vermieden werden müsse die Rückkehr zum therapeutischen „Chaos“. Neben randomisierten kontrollierten Studien und Meta-Analysen gibt es andere wichtige Instrumente und Methoden, die sich auch zur Validierung von Behandlungsverfahren bei seltenen Erkrankungen eignen. Individuelle Thera- pieversuche sollten methodisch sauber durchgeführt und sorgfältig dokumentiert werden.

\section{Resüimee}

Eine prägnante Zusammenfassung zur Therapie der primären Ziliendyskinesie fällt schwer. Dieser Experten-Workshop bestätigte, dass es kaum Originalarbeiten gibt, die eine bestimmte Behandlung begründen können. Insofern ist der betreuende Arzt häufig auf Analogieschlüsse von anderen Krankheitsbildern angewiesen.

Wie bei anderen seltenen Erkrankungen der Atemwege ist es sinnvoll, die betroffenen Patienten in spezialisierten Behandlungszentren zu betreuen. Dort ist auch eine interdisziplinäre Zusammenarbeit von pneumologisch ausgewiesenen Ärzten, Physiotherapeuten und Hals-Nasen-Ohrenärzten gegeben.

Zur allgemeinen Umgebungsprophylaxe für Kinder mit PCD gehört eine rauchfreie Wohnung. Jeder Patient sollte alle altersentsprechenden Impfungen erhalten und zusätzlich gegen Pneumokokken und jährlich gegen Influenza geimpft werden. Eine zentrale Rolle bei der Therapie spielten Sekretmobilisation und Sekretolyse. Dazu werden physiotherapeutische Techniken und Medikamente angewendet, wie man sie auch von anderen Atemwegserkrankungen kennt. Ob eine antientzündliche Dauertherapie sinnvoll ist, kann mit wissenschaftlicher Literatur nicht belegt werden. Dennoch werden inhalative Kortikosteroide im klinischen Alltag bei PCD häufig verordnet. Ähnlich ist die Situation hinsichtlich der Anwendung von topischen Antibiotika. Bei Infektion mit Pseudomonas aeruginosa können Inhalation mit Tobramycin sinnvoll sein, wenngleich ein Wirksamkeitsnachweis für Patienten mit PCD fehlt. Systemische Antibiotika sind bei eitrigen Infektionen der oberen Atemwege indiziert und können bei Exazerbationen der Lungenerkrankung erforderlich werden.

Angesichts der unzureichenden Datenlage äußerten die Teilnehmer des Workshops den Wunsch, zukünftig multizentrische Studien durchzuführen. In Anbetracht der geringen Patientenzahlen ist dies eine wichtige Möglichkeit, die Erkenntnisse zur Behandlung zu verbessern. Bei der Betreuung des einzelnen Patienten sollte jeder Arzt den Nutzen eines neu verordneten Medikamentes systematisch überprüfen, idealerweise mit dem Instrument der $\mathrm{n}=1$-Studie.

\section{Erläuterungen zu Tab. 2}

\section{Asymptomatischer Säugling mit frisch diagnostizierter PCD}

Im Rahmen der Umgebungsprophylaxe sollte die Wohnung der Familie rauchfrei gehalten werden. Als physiotherapeutische Methode erlernen die Eltern erste Drainagemethoden. Eine enge Kooperation mit einer Krankengymnastin mit Schwerpunkt Atemphysiotherapie ist angezeigt. Säuglinge, die nach neonatalem Atemnotsyndrom diagnostiziert worden sind, können von inhalativen Kortikosteroiden profitieren. Diese sollten über ein Dosieraerosol mit Spacer verabreicht werden. Kurzwirksame inhalative $\beta_{2}$-Sympathomimetika können im Einzelfall sinnvoll sein. 
2. Symptomatisches Kleinkind ohne pulmonale Folgeerkrankungen

Mit Unterstützung durch die Krankengymnastin sollte die Methode der Atemphysiotherapie gesucht werden, die am besten zu den individuellen Gegebenheiten passt. Zur Physiotherapie kommt in diesem Alter auch der Flutter infrage.

Inhalative Steroide sind in dieser Altersgruppe häufig angezeigt. Zur Sekretolyse wird orales N-Acetylcystein empfohlen, und zwar in einer Dosierung von $10-20 \mathrm{mg} / \mathrm{kg}$ und Tag. Andere Experten verabreichen orale Sekretolytika nur bei akuten Exazerbationen. Inhalative Antibiotika sind nicht indiziert. Allerdings kann je nach aktuellem bakteriologischen Befund und klinischer Symptomatik eine systemische antibiotische Behandlung sinnvoll sein, von der rund 30\% der Kinder profitieren. Für eine Langzeittherapie kann das Antibiotikum alle sechs Monate gewechselt werden. Dabei sollte man auch einen Auslassversuch (beispielsweise in den Sommermonaten) in Erwägung ziehen. Bronchodilatatoren sind klar angezeigt, wenn die Kinder im Broncholysetest davon profitieren. Auch langwirksame inhalative $\beta_{2}$-Sympathomimetika können in dieser Altersgruppe verordnet werden.

3. Symptomatischer Patient (Kind oder Jugendlicher) mit manifester Folgeerkrankung

Regelmäßige, konsequente Physiotherapie sowie die Inhalation mit Kortikosteroiden sind angezeigt. Auch systemische Steroide können im Einzelfall sinnvoll sein. Orales N-Acetylcystein wird regelmäßig verordnet. Dabei muss darauf geachtet werden, dass eine effiziente Physiotherapie erfolgt. Auch die Inhalation von Dornase alfa kann wirksam sein, wobei nach vier Wochen überprüft werden sollte, ob der Therapieversuch erfolgreich war.

Bei Nachweis von Pseudomonas aeruginosa kann eine inhalative Antibiotikatherapie erfolgen, alternativ die systemische Gabe von Antibiotika. Auch eine prophylaktische antimikrobielle Therapie wird bei vielen Patienten sinnvoll sein. Aus der Gruppe der Bronchodilatatoren können langwirksame inhalative $\beta_{2}$-Sympathomimetika zum Einsatz kommen.

\section{Akute Exazerbation}

Eine Physiotherapie sollte erst dann erfolgen, wenn sich der Patient wieder in einem stabilen Zustand befindet. Es muss vermieden werden, dass sich mobilisiertes Sekret in bestimmten Lungenabschnitten staut, weil es nicht genügend abgehustet werden kann. Eine Behandlung mit inhalativen Kortikosteroiden soll nicht abgesetzt werden. Bei schwerer Obstruktion können systemische Steroide indiziert sein. Zur Antibiotikatherapie muss auch die intravenöse Behandlung in Betracht gezogen werden.

\section{Literatur}

${ }^{1}$ Ahrens P, Körbler H. Primäre ziliäre Dyskinesie: Klinik, Diagnostik und Differentialdiagnostik. HNO aktuell 2002; 10: $391-400$

2 Meeks M, Bush A. Primary ciliary dyskinesia (PCD). Pediatr Pulmonol 2000; 29: 307-316

${ }^{3}$ Bush A, Cole P, Hariri M et al. Primary ciliary dyskinesia: diagnosis and standards of care. Eur Respir J 1998; 12: $982-988$

${ }^{4}$ Phillips GE, Thomas S, Heather S et al. Airway response of children with primary ciliary dyskinesia to exercise and $\beta_{2}$-agonist challenge. Eur Respir J 1998; 11: 1389-1391

${ }^{5}$ Heatley DG, McConnell KE, Kille TL et al. Nasal irrigation for the alleviation of sinonasal symptoms. Otolaryngol Head Neck Surg 2001; 125: $44-48$

${ }^{6}$ Taccariello M, Parikh A, Darby Y et al. Nasal douching as a valuable adjunct in the management of chronic rhinosinusitis. Rhinology 1999; 37: $29-32$

${ }^{7}$ Rabago D, Zgierska A, Mundt M et al. Efficacy of daily hypertonic saline nasal irrigation among patients with sinusitis: a randomized controlled trial. J Fam Pract 2002; 51: 1049- 1055

${ }^{8}$ Desrosiers MY, Salas-Prato M. Treatment of chronic rhinosinusitis refractory to other treatments with topical antibiotic therapy delivered by means of a large-particle nebulizer: results of a controlled trial. Otolaryngol Head Neck Surg 2001; 125: 265-269

${ }^{9}$ Shoseyov D, Bibi H, Shai P et al. Treatment with hypertonic saline versus normal saline nasal wash of pediatric chronic sinusitis. J Allergy Clin Immunol 1998; 101: 602-605

${ }^{10}$ Karadag B, James AJ, Gultekin E et al. Nasal and lower airway level of nitric oxide in children with primary ciliary dyskinesia. Eur Respir J 1999; 13: $1402-1405$

${ }^{11}$ Niggemann B. Ziliäre Dyskinesie. In: Rieger C, von der Hardt H, Sennhauser FH, Wahn U, Zach MS (Hrsg). Pädiatrische Pneumologie. Berlin, Heidelberg: Springer, 1999: 959-961

12 Hodson ME, Gallagher CG, Govan JR. A randomised clinical trial of nebulised tobramycin or colistin in cystic fibrosis. Eur Respir J 2002; 20: $658-664$

${ }^{13}$ Barker AF, Couch L, Fiel SB et al. Tobramycin solution for inhalation reduces sputum Pseudomonas aeruginosa density in bronchiectasis. Am J Respir Crit Care Med 2000; 162: 481-485

${ }^{14}$ Lin HC, Cheng HF, Wang CH et al. Inhaled gentamicin reduces airway neutrophil activity and mucus secretion in bronchiectasis. Am J Respir Crit Care Med 1997; 155: 2024-2029

15 Orriols R, Roig J, Ferrer J et al. Inhaled antibiotic therapy in non-cystic fibrosis patients with bronchiectasis and chronic bronchial infection by Pseudomonas aeruginosa. Respir Med 1999; 93: 476-480

16 O'Donnell AE, Barker AF, Ilowite JS et al. Treatment of idiopathic bronchiectasis with aerosolized recombinant human DNase I. rhDNase Study Group. Chest 1998; 113: 1329-1334

${ }_{17}$ Mandel EM, Casselbrant ML, Rockette HE et al. Efficacy of antimicrobial prophylaxis for recurrent middle ear effusion. Pediatr Infect Dis J 1996; 15: $1074-1082$

${ }^{18}$ Fliss DM, Dagan R, Houri Z et al. Medical management of chronic suppurative otitis media without cholesteatoma in children. J Pediatr 1990; 116: $991-996$

${ }^{19}$ Acuin J, Smith A, Mackenzie I. Interventions for chronic suppurative otitis media (Cochrane Review). Cochrane Library 2003

${ }^{20}$ Morris P, Leach A. Antibiotics for persistent nasal discharge (rhinosinusitis) in children (Cochrane Review). Cochrane Library 2003

${ }^{21}$ Sackett DL, Richardson WS, Rosenberg WMC et al. Evidence-Based medicine: how to practice and teach EBM. 2 ed. London: Churchill-Livingstone, 2000

22 Woodcock A, Forster L, Matthews E et al. Control of exposure to mite allergen and allergen-impermeable bed covers for adults with asthma. N Engl J Med 2003; 349: 225 - 236

${ }^{23}$ Tasche MJ, Uijen JH, Bernsen RM et al. Inhaled disodium cromoglycate (DSCG) as maintenance therapy in children with asthma: a systematic review. Thorax 2000; 55: 913-920

${ }^{24}$ Wainwright C, Altamirano L, Cheney M et al. A multicenter, randomized, double-blind, controlled trial of nebulized epinephrine in infants with acute bronchiolitis. N Engl J Med 2003; 349: 27 - 35

25 Gallin JI, Alling DW, Malech HL et al. Itraconazole to prevent fungal infections in chronic granulomatous disease. N Engl J Med 2003; 348 : $2416-2422$ 